\title{
A Novel Method Based on Oblique Projection Technology for Mixed Sources Estimation
}

\author{
Weijian Si, Xiaolin Li, Yilin Jiang, and Liangtian Wan \\ College of Information and Communication Engineering, Harbin Engineering University, Harbin 150001, China \\ Correspondence should be addressed to Yilin Jiang; jiangyilin@hrbeu.edu.cn
}

Received 14 July 2014; Revised 5 October 2014; Accepted 5 October 2014; Published 23 October 2014

Academic Editor: Massimo Scalia

Copyright (C) 2014 Weijian Si et al. This is an open access article distributed under the Creative Commons Attribution License, which permits unrestricted use, distribution, and reproduction in any medium, provided the original work is properly cited.

Reducing the computational complexity of the near-field sources and far-field sources localization algorithms has been considered as a serious problem in the field of array signal processing. A novel algorithm caring for mixed sources location estimation based on oblique projection is proposed in this paper. The sources are estimated at two different stages and the sensor noise power is estimated and eliminated from the covariance which improve the accuracy of the estimation of mixed sources. Using the idea of compress, the range information of near-field sources is obtained by searching the partial area instead of the whole Fresnel area which can reduce the processing time. Compared with the traditional algorithms, the proposed algorithm has the lower computation complexity and has the ability to solve the two closed-spaced sources with high resolution and accuracy. The duplication of range estimation is also avoided. Finally, simulation results are provided to demonstrate the performance of the proposed method.

\section{Introduction}

In the past few years, source location has received a significant amount of attention [1-3]. In practice, the nearfield and far-field sources coexist in most cases, and the conventional algorithms for the far-field sources always to estimate the parameters of the sources. For instance, the traditional MUSIC algorithm [4] and ESPRIT (Estimation of Signal Parameters via Rotational Invariance Techniques) algorithm [5] cannot solve the above situation. Some specific algorithms are presented for near-field sources [6-16], such as two-dimensional (2-D) MUSIC method [6], the maximum likelihood method [10], the path following method [11], and the polynomial rooting method [12]. The high-order ESPRIT method was presented in [13]. However, when dealing with the mixed sources, the performances of these algorithms would decline. The computational complexity will increase meanwhile. Thus the research issues of estimating for nearfield and far-field sources simultaneously have important practical significance and have gradually become a new hot spot.

Several algorithms for both the near-field and far-field signals which coexist are presented. Most of them are based on high-order cumulant. TSMUSIC (two-stage MUSIC) method [17] solves mixed signal by constructing two special cumulants, avoiding the estimation failure problem. However, the computational complexity increases as well. Similar to those existing methods aforementioned, the algorithm proposed in [18] is improved based on TSMUSIC. The highorder cumulant is constructed, and the DOA and range of mixed sources are obtained by reconstructing the sparse matrix. [19] is also improved based on TSMUSIC. Mixedorder MUSIC algorithm and a sparse symmetric array are used in [19]. However they use the high-order cumulant, thus suffering from burdensome computation as well. Moreover, the high-order cumulant-based algorithms would fail in the presence of Gaussian sources. And the paramount disadvantage of these methods is that they need large number of snapshots. Such simultaneous estimation influences the estimation performance of near-field sources.

According to the signal model, we know that the nearfield sources have two parameters to estimate, while the far-field sources only have one. It means that so far most literatures about mixed sources estimation estimate the range information of far-field sources which is no need. It not only increases the computation complexity but also reduces the estimation accuracy. In this paper, we consider that the farfield sources only need to estimate one parameter which is 
different from near-field sources. Two kinds of sources will be estimated separately. Meanwhile the algorithms aforementioned for near-field sources need to search the whole Fresnel area which increases the processing time. The proposed algorithm uses the idea of compress and obtains the distance information of near-field sources by searching the part of Fresnel area instead of the whole Fresnel area which can reduce the processing time.

The proposed algorithm includes four steps as follows: (1) utilize MUSIC algorithm to obtain the DOA of far-field sources and utilize the angle information of far-field sources to construct the oblique projector operation; (2) the sensor noise power is estimated and eliminated from the covariance. Meanwhile utilize the oblique projector operation to eliminate the information of far-field sources from covariance matrix and retain the information of near-field sources; (3) construct the polynomial by the symmetrical structure of the arrays and obtain the DOA of the near-field sources by solving the root of polynomial; (4) use the idea of compress and obtain the distance information of near-field sources which is obtained by searching the part of Fresnel area instead of the whole Fresnel area.

The rest of this paper is organized as follows. The mixed sources model including far-field sources and nearfield sources is introduced in Section 2. The estimation of far-field sources is shown in Section 3. The algorithm for near-field sources is shown in Section 4. The performance analysis about the proposed algorithm is given in Section 5. The discussion about the proposed algorithm is given in Section 6. Simulation results are presented in Section 7, and conclusion is in Section 8.

\section{Signal Model of Mixed Source}

As shown in Figure 1, $K$ narrowband sources, contained by $K_{n}$ near-field sources and $K_{f}$ far-field sources, are impinging on a symmetric uniform linear array with $2 N+1$ nondirectional sensors. The center of array is considered as phase reference point. The data received by array $i$ can be expressed as

$$
\begin{array}{r}
x_{i}(t)=\sum_{k=1}^{K_{n}} a_{n_{i}}\left(\theta_{k}, r_{k}\right) s_{n_{k}}(t)+\sum_{k=1}^{K_{f}} a_{f_{i}}\left(\theta_{k}\right) s_{f_{k}}(t)+n_{i}(t) \\
-N \leq i \leq N,
\end{array}
$$

where $s_{f_{k}}(t)$ is the $k$ th narrowband far-field source, $s_{n_{k}}(t)$ is the $k$ th narrowband near-field source, and $n_{i}(t)$ is the additive Gaussian noise received by the $i$ th array.

The vector form of data received by array can be expressed as

$$
\mathbf{x}(t)=\mathbf{A}_{n}(\theta, r) \mathbf{s}_{n}(t)+\mathbf{A}_{f}(\theta) \mathbf{s}_{f}(t)+\mathbf{n}(t),
$$

where $\mathbf{s}_{n}(t)=\left[s_{n_{1}}(t), \ldots, s_{n_{K_{n}}}(t)\right]^{T}$ is a $K_{n} \times 1$ vector of nearfield sources; $\mathbf{s}_{f}(t)=\left[s_{f_{1}}(t), \ldots, s_{f_{K_{f}}}(t)\right]^{T}$ is a $K_{f} \times 1$ vector of far-field sources; $\mathbf{n}(t)$ is the $2 N+1$ additive complex Gaussian white noise vector.

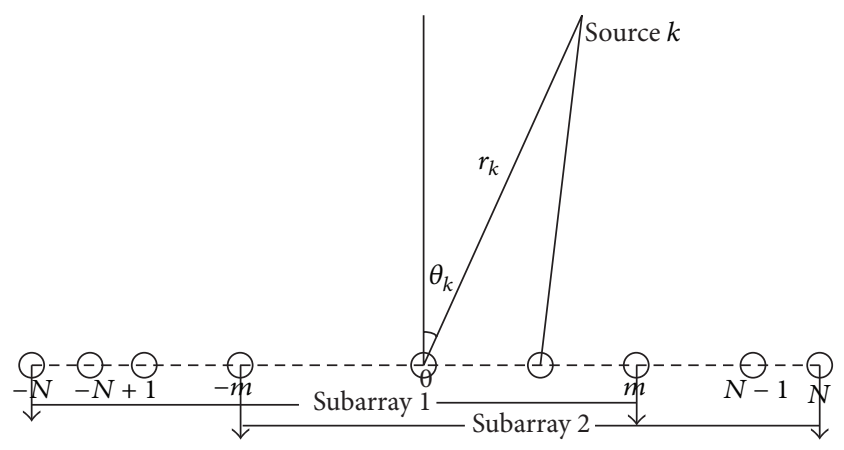

FIGURE 1: Symmetric uniform linear array configuration.

$\mathbf{A}_{n}(\theta, r)$ and $\mathbf{A}_{f}(\theta)$ represent the near-field and the farfield sources manifold matrix separately, which are expressed as

$$
\begin{gathered}
\mathbf{A}_{n}(\theta, r)=\left[\mathbf{a}_{n}\left(\theta_{1}, r_{1}\right), \ldots, \mathbf{a}_{n}\left(\theta_{K_{n}}, r_{K_{n}}\right)\right] \\
\mathbf{A}_{f}(\theta)=\left[\mathbf{a}_{f}\left(\theta_{1}\right), \ldots, \mathbf{a}_{f}\left(\theta_{K_{f}}\right)\right], \\
\mathbf{a}_{n}\left(\theta_{k}, r_{k}\right)=\left[\exp \left(j\left(-N \omega_{k}+N^{2} \phi_{k}\right)\right), \ldots,\right. \\
\left.\exp \left(j\left(N \omega_{k}+N^{2} \phi_{k}\right)\right)\right]^{T} \\
\mathbf{a}_{f}\left(\theta_{k}\right)=\left[\exp \left(-j N \omega_{k}\right), \ldots, \exp \left(j N \omega_{k}\right)\right]^{T}
\end{gathered}
$$

and $\omega_{k}, \phi_{k}$ are

$$
\begin{gathered}
\omega_{k}=-\frac{2 \pi d \sin \left(\theta_{k}\right)}{\lambda} \\
\phi_{k}=\frac{\pi d^{2} \cos \left(\theta_{k}\right)^{2}}{\lambda r_{k}},
\end{gathered}
$$

where $\theta_{k}$ and $r_{k} k=\left[1,2, \ldots, K_{n}\right]$ are the DOA and range of the $k$ th near-field source, respectively, and $\theta_{k} k=$ $\left[1,2, \ldots, K_{f}\right]$ are the DOA of $k$ th far-field source, respectively.

In this paper, we make the assumptions as follows:

(1) the signals are statistically independent;

(2) the sensor noise is the additive white Gaussian noise, which is independent of the signal sources;

(3) the antenna spacing $d$ is within a quarter-wavelength.

\section{DOA Estimation for Far-Field Sources}

According to (2), these received data can be expressed in another way:

$$
\mathbf{x}(t)=\left[\begin{array}{ll}
\mathbf{A}_{f} & \mathbf{A}_{n}
\end{array}\right]\left[\mathbf{s}_{f}(t) \mathbf{s}_{n}(t)\right]^{T}+\mathbf{n}(t)=\widehat{\mathbf{A}} \widehat{\mathbf{s}}(t)+\mathbf{n}(t) .
$$

Collect $M$ snapshots and define $\mathbf{X}=[\mathbf{x}(1), \mathbf{x}(2), \ldots$, $\mathbf{x}(M)$ ], which is denoted as

$$
\mathbf{X}=\widehat{\mathbf{A}} \widehat{\mathbf{S}}+\mathbf{N}
$$


where $\widehat{\mathbf{S}} \in \mathbf{C}^{K \times M}$ is the source matrix for $M$ samples and $\mathbf{N} \epsilon$ $\mathrm{C}^{K \times 2 N+1}$ is the noise matrix.

The covariance matrix $\mathbf{R}$ received by the array can be estimated with $M$ snapshots by

$$
\begin{aligned}
\mathbf{R} & =E\left[\mathbf{X} \mathbf{X}^{H}\right]=\widehat{\mathbf{A}} \mathbf{R}_{S} \widehat{\mathbf{A}}^{H}+\sigma^{2} \mathbf{I} \\
& =\mathbf{A}_{f} \mathbf{R}_{f} \mathbf{A}_{f}^{H}+\mathbf{A}_{n} \mathbf{R}_{n} \mathbf{A}_{n}^{H}+\sigma^{2} \mathbf{I} \\
& =\mathbf{U}_{S} \boldsymbol{\Lambda}_{S} \mathbf{U}_{S}^{H}+\mathbf{U}_{N} \boldsymbol{\Lambda}_{N} \mathbf{U}_{N}^{H},
\end{aligned}
$$

where $\mathbf{U}_{S}$ represents the eigenvectors corresponding to the $K$ large values. $\mathbf{U}_{N}$ represent the eigenvectors corresponding to the last small values.

In this paper, the DOAs for far-field sources are estimated by MUSIC algorithm.

Spatial spectrum function is constructed as follows:

$$
P_{\text {MUSIC }}=\frac{1}{\mathbf{a}_{f}^{H}(\theta) \mathbf{U}_{N} \mathbf{U}_{N}^{H} \mathbf{a}_{f}(\theta)},
$$

where

$$
\begin{aligned}
\mathbf{a}_{f}(\theta)= & {\left[\exp \left(j\left(\frac{2 \pi N d \sin (\theta)}{\lambda}\right)\right), \ldots,\right.} \\
& \left.\exp \left(-j\left(\frac{2 \pi N d \sin (\theta)}{\lambda}\right)\right)\right]^{T} .
\end{aligned}
$$

Continuously changing the value of $\theta$ to search spectral spectrum peak, the DOA estimation for far-field sources will be obtained, but the DOA estimation for near-field sources cannot be obtained in this processing, as the manifold vectors of the near-field and the far-field source are described by different equations. They belong to two different manifold curves within the same observation space. When using this manifold vector of far-field sources to search spectrum peak, it will not form the peaks in near-field sources position. Thus in this method we can only obtain the DOA estimation for far-field sources.

\section{DOA Estimation for Near-Field Sources}

In order to estimate DOA for near-field source separately, we need to remove far-field source from the received data covariance matrix. According to the oblique projection operation's characteristics, we can use oblique projection operation to extract near-field source from the data covariance matrix.

Our aim is to retain the near-field sources corresponding information $\mathbf{A}_{n}$ and eliminate far-field sources corresponding information $\mathbf{A}_{f}$. Therefore, we can first obtain projection operator $\mathbf{E}_{\mathbf{A}_{f} \mathbf{A}_{n}}$ just containing the far-field sources $\mathbf{A}_{f}$ and then obtain the information of near field source by subtracting the information of far-field sources.

Utilizing the character of oblique projection operator, we will structure oblique projection operator $\mathbf{E}_{\mathbf{A}_{f} \mathbf{A}_{n}}$ satisfying

$$
\mathbf{E}_{\mathbf{A}_{f} \mathbf{A}_{n}}=\mathbf{A}_{f}\left(\mathbf{A}_{f}^{H} \mathbf{P}_{\mathbf{A}_{n}}^{\perp} \mathbf{A}_{f}\right)^{-1} \mathbf{A}_{f}^{H} \mathbf{P}_{\mathbf{A}_{n}}^{\perp} .
$$

However, $\mathbf{P}_{\mathbf{A}_{n}}^{\perp}$ of (10) needs the steer vector $\mathbf{A}_{n}$ of the near-field sources to construct it. In other words, it needs the information of near-field sources. Thus it cannot be realized. According to the literature [20], when $K<(2 N+1), \mathbf{E}_{\mathbf{A}_{f} \mathbf{A}_{n}}$ can be expressed as

$$
\mathbf{E}_{\mathbf{A}_{f} \mathbf{A}_{n}}=\mathbf{A}_{f}\left(\mathbf{A}_{f}^{H} \mathbf{R}^{\#} \mathbf{A}_{f}\right)^{-1} \mathbf{A}_{f}^{H} \mathbf{R}^{\#},
$$

where \# means pseudoinverse.

According to the character of oblique projection operation, we have

$$
\mathbf{E}_{\mathbf{A}_{f} \mathbf{A}_{n}} \widehat{\mathbf{A}}=\mathbf{E}_{\mathbf{A}_{f} \mathbf{A}_{n}}\left[\begin{array}{ll}
\mathbf{A}_{f} & \mathbf{A}_{n}
\end{array}\right]=\left[\begin{array}{ll}
\mathbf{A}_{f} & \mathbf{0}
\end{array}\right] .
$$

Thus, we can obtain the covariance matrix $\mathbf{R}_{n}$ of near-field sources

$$
\mathbf{R}_{n}=\left(\mathbf{I}_{M}-\mathbf{E}_{\mathbf{A}_{f} \mathbf{A}_{n}}\right)\left(\mathbf{R}-\widehat{\sigma}^{2} \mathbf{I}\right)\left(\mathbf{I}_{M}-\mathbf{E}_{\mathbf{A}_{f} \mathbf{A}_{n}}\right)^{H}=\mathbf{A}_{n} \mathbf{R}_{S_{n}} \mathbf{A}_{n}^{H},
$$

where $\widehat{\sigma}^{2}$ is the estimation of sensor noise power and it is obtained from the mean of the $2 N+1-K$ smallest eigenvalues of $\mathbf{R}$ [21]:

$$
\widehat{\sigma}^{2}=\frac{1}{2 N+1-K} \sum_{i=K+1}^{2 N+1} \lambda_{N i}
$$

where $\lambda_{N i}$ is the last small eigenvalues of $\mathbf{R}$.

4.1. DOA Estimation of the Near-Field Sources. Now we use the new matrix $\mathbf{R}_{n}$ which only contains the information of near-field signals to estimate the direction and the range information of near-field sources. Eigenvalue decomposition function (12) can be obtained as follows:

$$
\mathbf{R}_{n}=\mathbf{U}_{c} \boldsymbol{\Lambda}_{c} \mathbf{U}_{c}^{H}=\mathbf{U}_{N n} \boldsymbol{\Lambda}_{N n} \mathbf{U}_{N n}^{H}+\mathbf{U}_{S n} \Lambda_{S n} \mathbf{U}_{S n}^{H},
$$

where $\mathbf{U}_{N n} \in C^{(2 N+1) \times\left(2 N+1-K_{n}\right)}$ represents the eigenvectors corresponding to the $2 N+1-K_{n}$ small values spanning the noise subspace of $\mathbf{R}_{n} ; \mathbf{U}_{S n} \in C^{(2 N+1) \times K_{n}}$ represents the eigenvectors corresponding to the $K_{n}$ large values spanning the signal subspace of $\mathbf{R}_{n}$. Thus $\mathbf{U}_{S n}$ should be given as a linear combination of all array response vectors; then we can rewrite $\mathbf{U}_{S n}$ as $\mathbf{A}_{n} \mathbf{G}$ :

$$
\mathbf{U}_{S n}=\mathbf{A}_{n} \mathbf{G}
$$

where $\mathbf{G}$ is a full-rank matrix

The literature [15] divides the ULA into two subarrays as shown in Figure 1. The first subarray is formed with the first $N+m+1$ sensors in ascending order (from sensor $-N$ to $m$ ), and the second subarray is formed with the last $N+m+1$ sensors in descending order (from sensors $N$ to sensors $-m$ ). In this paper, $m$ is defined as 0 ; the signal subspace of the two subarrays can be written as

$$
\mathbf{A}_{n} \mathbf{G}=\left[\begin{array}{c}
\mathbf{A}_{n 1} \mathbf{G} \\
\text { the last } N \text { rows }
\end{array}\right]=\left[\begin{array}{c}
\text { the first } N \text { rows } \\
\mathbf{J A}_{n 2} \mathbf{G}
\end{array}\right] .
$$


$\mathbf{U}_{S n}$ can be rewrite as

$$
\mathbf{U}_{S n}=\left[\begin{array}{c}
\mathbf{U}_{S n 1} \\
\text { the last } N \text { rows }
\end{array}\right]=\left[\begin{array}{c}
\text { the first } N \text { rows } \\
\mathbf{U}_{S n 2}
\end{array}\right] \text {, }
$$

where $\mathbf{A}_{n 1}$ and $\mathbf{A}_{n 2}$ represent the steering vector of subarrays 1 and 2 , respectively. $\mathbf{U}_{S n 1}$ and $\mathbf{U}_{S n 2}$ represent the signal subspace of subarrays 1 and 2, respectively.

The algorithm proposed in [15] utilizes the symmetric antenna structure to construct the spectrum function as follows:

$$
\begin{aligned}
\mathbf{P}_{\text {MUSIC }}= & \\
& \times\left(\operatorname { d e t } \left(\left[\left(\mathbf{D}\left(\theta_{1}\right)-\psi(\theta)\right) \mathbf{a}_{n 1}\left(\theta_{1}, r_{1}\right) \cdots\right.\right.\right. \\
& \left.\left.\left.\quad\left(\mathbf{D}\left(\theta_{K_{n}}\right)-\psi(\theta)\right) \mathbf{a}_{n 1}\left(\theta_{K_{n}}, r_{K_{n}}\right)\right] \mathbf{G}\right)\right)^{-1},
\end{aligned}
$$

where $\mathbf{D}\left(\theta_{k}\right)=\operatorname{diag}\left[\exp \left(-j\left(4 \pi \sin \left(\theta_{k}\right) N / \lambda\right)\right), \ldots, 1\right]$; the form of $\psi(\theta)$ is similar to $\mathbf{D}\left(\theta_{k}\right)$; the difference here is that $\theta$ of $\psi(\theta)$ is unknown:

$$
\psi(\theta)=\operatorname{diag}\left[\exp \left(-j\left(\frac{4 \pi \sin (\theta) N}{\lambda}\right)\right), \ldots, 1\right] .
$$

In order to reduce the computational complexity, the proposed algorithm uses the polynomial instead of the spectrum peak search. The constructed polynomial is shown as

$$
\begin{aligned}
f(z)=\operatorname{det}\left(\mathbf{W}^{H} \mathbf{J} \mathbf{U}_{S 2}-\mathbf{W}^{H} \mathbf{P}(z) \mathbf{U}_{S 1}\right) \\
=\operatorname{det}\left(\mathbf { W } ^ { H } \left[\left(\mathbf{D}\left(\theta_{1}\right)-\mathbf{P}(z)\right) \mathbf{a}_{n 1}\left(\theta_{1}, r_{1}\right), \ldots,\right.\right. \\
\left.\left.\quad\left(\mathbf{D}\left(\theta_{K_{n}}\right)-\mathbf{P}(z)\right) \mathbf{a}_{n 1}\left(\theta_{K_{n}}, r_{K_{n}}\right)\right] \mathbf{G}\right),
\end{aligned}
$$

where $\mathbf{P}(z)=\operatorname{diag}\left(\left[1, z, \ldots, z^{K_{n}}\right]^{T}\right)$.

When $z=\exp \left(-j\left(4 \pi d \sin \left(\theta_{k}\right) / \lambda\right)\right), f(z)$ will become zero.

According to calculating roots of $f(z)$ method, we get the DOA of the near-field sources.

Noting order of the polynomial $f(z)$ is $2(N-1)$, which is together with $(N-1)$ pairs of roots, among them each pair of roots has conjugate relationship, and $2 K_{n}$ roots are being distributed on the whole circle:

$$
z=\exp \left(-j\left(\frac{4 \pi d \sin \left(\theta_{k}\right)}{\lambda}\right)\right) \quad 1 \leq k \leq K_{n} .
$$

It satisfies (19) when knowing the covariance matrix of this case accurately, while in practical application, only $K_{n}$ roots approximately lay on the unit circle; unfortunately the existing error in the estimated covariance matrix cannot be avoided through the limited snapshot number:

$$
\theta_{k}=\arcsin \left(-\frac{\lambda \arg (z)}{4 \pi d}\right) \quad 1 \leq k \leq K_{n}
$$

The DOA estimation of near-field sources is obtained by the above method.

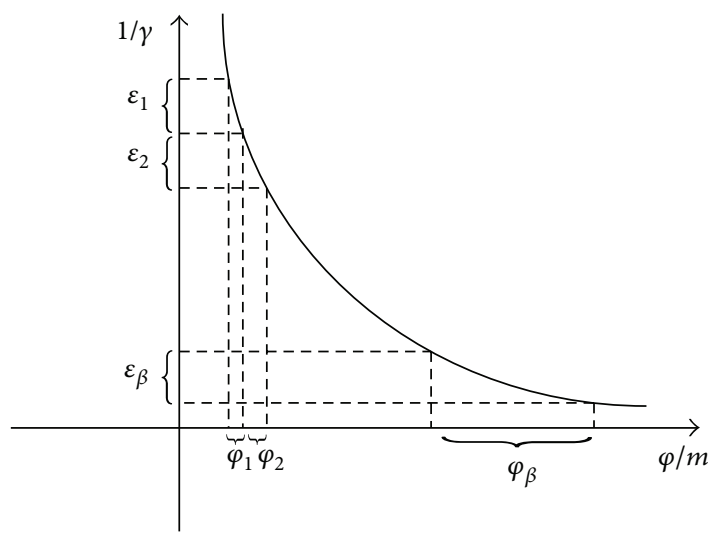

FIGURE 2: Spatial range dividing, where the length of the $i$ th range sector in $m=1 / r$ space is $\varepsilon_{i}\left(\varepsilon_{i}=\kappa / \beta=\varepsilon\right)$ and the length in $r$ space is $\varphi=\left[\varphi_{1}, \varphi_{2}, \ldots, \varphi_{\beta}\right]$.

4.2. Range Estimation of Near-Field Sources. After getting the DOA of near field sources, the traditional algorithms often substitute the angle information into steer vectors to estimate range information. This idea can avoid the parameter pair problem. But it needs to search in the whole area, leading to increase of the computation. The algorithm in this paper just searches part of the area instead of the whole area, reducing the complexity.

In order to reduce the computational complexity, the total range field-of-view is divided into $\beta$ small sectors $\varphi_{i}, i=$ $1,2, \ldots, \beta$, which is shown in Figure 2 :

$$
\varphi=\left[\varphi_{1}, \varphi_{2}, \ldots, \varphi_{\beta}\right] .
$$

Here, the length of each section in the inverse space is given by

$$
\varepsilon_{1}=\varepsilon_{2}=\varepsilon_{3} \cdots \varepsilon_{\beta}=\frac{\kappa}{\beta}=\varepsilon,
$$

where $\kappa=\left(1 / 0.62\left(r^{3} / \lambda\right)^{0.5}-1 / 2\left(r^{2} / \lambda\right)\right)$.

It can be seen from Figure 2 that $\forall r_{m} \in \varphi_{m}, m=1,2, \ldots$, $\beta$, and there exists $\forall r_{k} \in \varphi_{k}, m=1,2, \ldots, \beta, k \neq m$ satisfying

$$
\frac{1}{r_{m}}=\frac{1}{r_{k}}+(k-m) \varepsilon \text {. }
$$

Let $a_{n_{p}}\left(\theta_{m}, r_{m}\right), p=1,2, \ldots, 2 N+1$ denote the $p$ th element of $\mathbf{a}_{n}(\theta, r)$; then

$$
\begin{aligned}
& a_{n_{p}}\left(\theta_{m}, r_{m}\right) \\
&= \exp \left(\frac{-j 2 \pi d p \sin \left(\theta_{m}\right)}{\lambda}+\frac{j \pi d^{2} p^{2} \cos \left(\theta_{m}\right)^{2}}{\lambda r_{m}}\right) \\
&=\exp \left(\frac{-j 2 \pi d p \sin \left(\theta_{m}\right)}{\lambda}+\frac{j \pi d^{2} p^{2} \cos \left(\theta_{m}\right)^{2}}{\lambda}\right. \\
&\left.\quad \times\left(\frac{1}{r_{k}}+(k-m) \varepsilon\right)\right)
\end{aligned}
$$




$$
\begin{aligned}
= & \exp \left(\frac{-j 2 \pi d p \sin \left(\theta_{m}\right)}{\lambda}+\frac{j \pi d^{2} p^{2} \cos \left(\theta_{m}\right)^{2}}{\lambda r_{k}}\right) \\
& \cdot \exp \left(\frac{j \pi d^{2} p^{2} \cos \left(\theta_{m}\right)^{2}}{\lambda}(k-m) \varepsilon\right) \\
= & \chi_{k, p} a_{n_{p}}\left(\theta_{m}, r_{k}\right),
\end{aligned}
$$

where $\chi_{k, p}$ is a constant given by

$$
\chi_{k, p}=\exp \left(\frac{j \pi d^{2} p^{2} \cos \left(\theta_{m}\right)^{2}}{\lambda}(k-m) \varepsilon\right) .
$$

Noting that $\varepsilon$ and $s$ are fixed here, $\chi_{k, p}$ only depends on $k$ and $p$. It is implied by (27) that

$$
\begin{aligned}
\mathbf{a}_{n}\left(\theta_{m}, r_{m}\right)= & {\left[\chi_{k, 0} a_{n_{1}}\left(\theta_{m}, r_{k}\right), \ldots, \chi_{k, 2 N+1} a_{n_{2 N+1}}\left(\theta_{m}, r_{k}\right)\right]^{T} } \\
= & {\left[\chi_{k, 0}, \ldots, \chi_{k, 2 N+1}\right] } \\
& \odot\left[a_{n_{1}}\left(\theta_{m}, r_{k}\right), \ldots, a_{n_{2 N+1}}\left(\theta_{m}, r_{k}\right)\right]^{T} \\
= & \chi_{k} \odot \mathbf{a}_{n}\left(\theta_{m}, r_{k}\right),
\end{aligned}
$$

where $\odot$ stands for Hadamard product and $\chi_{k}$ is a $(2 N+1) \times 1$ vector which is defined as

$$
\chi_{k}=\left[\chi_{k, 0}, \chi_{k, 1}, \ldots, \chi_{k, 2 N+1}\right]^{T} .
$$

Assuming that $r_{m} \in \varphi_{m}, m=1,2, \ldots, \beta$ is the range information of the $m$ th source and according to the orthogonality between the steering vector and the noise subspace, we have

$$
\mathbf{a}_{n}\left(\theta_{m}, r_{m}\right)^{H} \mathbf{u}_{i}=0, \quad i=1,2, \ldots,\left(2 N+1-K_{n}\right),
$$

where $\mathbf{u}_{i}$ is the $i$ th column of noise subspace $\mathbf{U}_{N n}$.

Substituting (29) into (30), we have

$$
\begin{aligned}
\mathbf{a}_{n}\left(\theta_{m}, r_{m}\right)^{H} \mathbf{u}_{i} & =\left(\boldsymbol{\chi}_{k} \odot \mathbf{a}_{n}\left(\theta_{m}, r_{k}\right)\right)^{H} \mathbf{u}_{i} \\
& =\left(\left(\mathbf{a}_{n}\left(\theta_{m}, r_{k}\right)^{*}\right)^{T} \odot\left(\chi_{k}^{*}\right)^{T}\right) \mathbf{u}_{i} \\
& =\mathbf{a}_{n}\left(\theta_{m}, r_{k}\right)^{H}\left(\left(\chi_{k}^{*}\right) \odot \mathbf{u}_{i}\right)=0 .
\end{aligned}
$$

$\mathbf{u}_{i, k}=\boldsymbol{\chi}_{k}^{*} \odot \mathbf{u}_{i}$ is the $i$ th column of noise subspace $\mathbf{U}_{k}$ :

$$
\begin{aligned}
\mathbf{U}_{k} & =\left[\mathbf{u}_{1, k}, \mathbf{u}_{2, k}, \ldots, \mathbf{u}_{\left(2 N+1-K_{n}\right), k}\right] \\
& =\left[\boldsymbol{\chi}_{k}^{*} \odot \mathbf{u}_{1}, \boldsymbol{\chi}_{k}^{*} \odot \mathbf{u}_{2}, \ldots, \boldsymbol{\chi}_{k}^{*} \odot \mathbf{u}_{\left(2 N+1-K_{n}\right)}\right] \\
& =\left[\frac{\boldsymbol{\chi}_{k}^{*}, \boldsymbol{\chi}_{k}^{*}, \ldots, \boldsymbol{\chi}_{k}^{*}}{\left(2 N+1-K_{n}\right)}\right] \odot\left[\mathbf{u}_{1}, \mathbf{u}_{2}, \ldots, \mathbf{u}_{\left(2 N+1-K_{n}\right)}\right] .
\end{aligned}
$$

In order to facilitate the derivation, let $\mathbf{U}_{1}=\mathbf{U}_{N n}$.

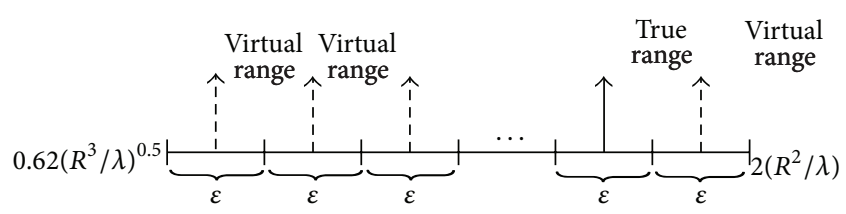

FIGURE 3: The virtual range information by the proposed algorithm.

Therefore, $\mathbf{U}_{k}, k=1,2, \ldots, \beta$ is called noise-like subspace cluster (NLSC), and the intersection of NLSC is defined by

$$
\operatorname{span}\left(\mathbf{U}_{\text {new }}\right)=\cap \operatorname{span}\left(\mathbf{U}_{k}\right) .
$$

It can be seen from (30) that $\mathbf{a}_{n}\left(\theta_{m}, r_{m}\right) \perp \operatorname{span}\left(\mathbf{U}_{1}\right)$ is equivalent to $\mathbf{a}_{n}\left(\theta_{m}, r_{k}\right) \perp \operatorname{span}\left(\mathbf{U}_{k}\right)$, where $r_{k} \in \varphi_{k}$, $k=1,2, \ldots, \beta, k \neq m$. Since $\mathbf{U}_{\text {new }}$ is the intersection of $\mathbf{U}_{k}, k=1,2, \ldots, \beta$, and $\operatorname{span}\left(\mathbf{U}_{\text {new }}\right)=\cap \operatorname{span}\left(\mathbf{U}_{k}\right)$, we have

$$
\mathbf{a}_{n}\left(\theta_{m}, r_{k}\right) \perp \operatorname{span}\left(\mathbf{U}_{\text {new }}\right), \quad k=1,2, \ldots, \beta .
$$

Based on such multiple orthogonal between $\mathbf{a}\left(\theta_{m}, r_{k}\right)$ and $\operatorname{span}\left(\mathbf{U}_{\text {new }}\right)$, a new spectrum function is defined as follows:

$$
\begin{aligned}
\max _{r} P_{\text {new }}(r) & =\frac{1}{\mathbf{a}_{n}^{H}\left(\theta_{m}, r\right) \widehat{\mathbf{U}}_{\text {new }} \widehat{\mathbf{U}}_{\text {new }}^{H} \mathbf{a}_{n}\left(\theta_{m}, r\right)} \\
& =\frac{1}{\left\|\widehat{\mathbf{U}}_{\text {new }}^{H} \mathbf{a}_{n}\left(\theta_{m}, r\right)\right\|} .
\end{aligned}
$$

It can be seen clearly from (35) that, for each true ROA (range of arrive) $r$, there exists one spectrum peak by the proposed algorithm in each range sector simultaneously, where $\beta-1$ virtual ROA are given by $r_{k}, k=1,2, \ldots, \beta, k \neq m$; this means that, for each true source, the spatial spectrum of the proposed algorithm generates $\beta-1$ is equivalent to virtual sources, which is shown in Figure 3. Traditional algorithms search on the whole Fresnel area. While the proposed algorithm only needs search on the part of Fresnel area to get the range information $r_{1}$ and then, using (26), to calculate the other range information $r_{2}, r_{3}, \ldots, r_{\beta}$. Since the steering vector $\mathbf{a}\left(\theta_{m}, r\right)$ is orthogonal to the original noise subspace only at the range of the true incident range information $r_{i}$, we can select the true range information $r_{i}$ among the $r_{1}, r_{2}, r_{3}, \ldots, r_{\beta}$ by minimizing $1 /\left\|\widehat{\mathbf{U}}_{N n}^{H} \mathbf{a}_{n}\left(\theta_{m}, r\right)\right\|$.

How to get the intersection of NLSC $\mathbf{U}_{\text {new }}$ is the core steps of the proposed algorithm. The specific process is given as follows.

Before constructing the intersection of NLSC $\mathbf{U}_{\text {new }}$, we introduce the intersection of SLSC (signal-like subspace cluster) $\mathbf{L}_{\text {new }}$ as an intermediate variable

$$
\begin{aligned}
\mathbf{L}_{k} & =\left[\mathbf{l}_{1, k}, \mathbf{l}_{2, k}, \ldots, \mathbf{l}_{K_{n}, k}\right] \\
& =\left[\chi_{k}^{*} \odot \mathbf{l}_{1}, \boldsymbol{\chi}_{k}^{*} \odot \mathbf{l}_{2}, \ldots, \boldsymbol{\chi}_{k}^{*} \odot \mathbf{l}_{K_{n}}\right] \\
& =\left[\frac{\boldsymbol{\chi}_{k}^{*}, \boldsymbol{\chi}_{k}^{*}, \ldots, \boldsymbol{\chi}_{k}^{*}}{K_{n}}\right] \odot\left[\mathbf{l}_{1}, \mathbf{l}_{2}, \ldots, \mathbf{l}_{K_{n}}\right] \quad k \in[1, \ldots, \beta] .
\end{aligned}
$$

Proof. See Appendix A. 
Unlike the algorithm proposed in [22] which uses the noise subspace to construct the new matrix $\mathbf{U}_{\text {new }}$, the proposed algorithm here in this paper adopts the signal subspace to construct the new matrix $\mathbf{U}_{\text {new }}$. The advantage of it is that it can reduce the computational complexity. Since the dimensions of signal subspace are much smaller than that of noise subspace [23].

The steps of getting NLSC $\mathbf{U}_{\text {new }}$ are summarized as follows.

(1) Construct the orthogonal matrix of signal subspace as follows:

$$
\mathbf{T}_{k}=\mathbf{L}_{k}\left(\mathbf{L}_{k}^{H} \mathbf{L}_{k}\right)^{-1} \mathbf{L}_{k}^{H}=\mathbf{L}_{k} \mathbf{L}_{k}^{H} .
$$

(2) Use $\mathbf{T}_{k}, k=1,2, \ldots, \beta$ to define the following matrix Q:

$$
\mathbf{Q}=\sum_{k=1}^{\beta} \mathbf{T}_{k}
$$

whose null space is $\operatorname{Null}(\mathbf{Q})=\left\{\delta / \delta \in C^{M}, \mathbf{Q} \delta=0\right\}$.

Then we have

$$
\operatorname{span}\left(\mathbf{U}_{\text {new }}\right)=\operatorname{Null}(\mathbf{Q}) .
$$

Proof. See Appendix B.

Therefore, we can use SVD to get the orthogonal basis for $\operatorname{Null}(\mathbf{Q})$, also giving an orthogonal basis for the intersection of NLSC equivalently. The SVD of $\mathbf{Q}$ can be shown as

$$
\mathbf{Q}=\Pi \mathbf{\Omega} \mathbf{\Xi}^{H}
$$

where $\boldsymbol{\Pi}=\left[\boldsymbol{\pi}_{1}, \boldsymbol{\pi}_{2}, \ldots, \boldsymbol{\pi}_{2 N+1}\right]$ and $\boldsymbol{\Xi}=\left[\boldsymbol{\xi}_{1}, \boldsymbol{\xi}_{2}, \ldots, \boldsymbol{\xi}_{2 N+1}\right]$ are the left and right singular matrix of $\mathbf{Q}$, respectively. $\boldsymbol{\Omega}=\operatorname{diag}\left(\tau_{1}, \tau_{2}, \ldots, \tau_{2 N+1}\right)$ is a diagonal matrix and $\tau_{i}$ is the $i$ th singular value of $\mathbf{Q}$. The proposed algorithm has $\beta K_{n}$ equivalent sources; there must exist $\beta K_{n}$ larger and $2 N+1-$ $\beta K_{n}$ smaller diagonal elements in $\Omega$. Hence $\tau_{i}$ can be sorted as

$$
\tau_{1} \geq \tau_{2} \geq \cdots \geq \tau_{\beta K_{n}}>\tau_{\beta K_{n}+1}=\cdots \tau_{2 N+1}=0 .
$$

Therefore, (41) can be rewritten as

$$
\mathbf{Q}=\Pi_{s} \boldsymbol{\Omega}_{s} \Xi_{s}^{H}+\Pi_{n} \boldsymbol{\Omega}_{n} \boldsymbol{\Xi}_{n}^{H},
$$

where

$$
\begin{gathered}
\Pi_{s}=\left[\boldsymbol{\pi}_{1}, \boldsymbol{\pi}_{2}, \ldots, \boldsymbol{\pi}_{\beta K_{n}}\right], \\
\boldsymbol{\Pi}_{n}=\left[\boldsymbol{\pi}_{\beta K_{n}+1}, \boldsymbol{\pi}_{\beta K_{n}+2}, \ldots, \boldsymbol{\pi}_{2 N+1}\right], \\
\boldsymbol{\Xi}_{s}=\left[\boldsymbol{\xi}_{1}, \boldsymbol{\xi}_{2}, \ldots, \boldsymbol{\xi}_{\beta K_{n}}\right], \\
\boldsymbol{\Xi}_{n}=\left[\boldsymbol{\xi}_{\beta K_{n}+1}, \boldsymbol{\xi}_{\beta K_{n}+2}, \ldots, \boldsymbol{\xi}_{2 N+1}\right] .
\end{gathered}
$$

$\xi_{i}, i=\beta K_{n}+1, \ldots, 2 N+1$ are associated with the zero singular values of $\mathbf{Q}$. According to the relationship between the singular values and singular vector, we have

$$
\mathbf{Q} \xi_{i}=0 \xi_{i} \quad i=\beta K_{n}+1, \ldots, 2 N+1 .
$$

Therefore, $\xi_{i}, i=\beta K_{n}+1, \ldots, 2 N+1$ are linearly independent satisfying $\xi_{i} \in \mathbf{C}^{2 N+1}, \mathbf{Q} \xi_{i}=0$. Hence, these vectors offer an orthogonal basis for $\operatorname{Null}(\mathbf{Q})$. According to (41), we have

$$
\mathbf{U}_{\text {new }}=\left(\boldsymbol{\xi}_{\beta K_{n}+1}, \boldsymbol{\xi}_{\beta K_{n}+2}, \ldots, \boldsymbol{\xi}_{2 N+1}\right)=\boldsymbol{\Xi}_{n}
$$

The steps of the proposed algorithm can be described as follows.

Step 1. Use (8) to construct the spatial spectrum function $P_{\text {MUSIC }}$, searching spectral spectrum peak, to get the DOA of far-field sources.

Step 2. Based on the information of far-field sources which is obtained, use (11) to construct the oblique project operator $\mathbf{E}_{\mathbf{A}_{f} \mathbf{A}_{n}}$.

Step 3. Utilize (13) to obtain the $\mathbf{R}_{n}$ only containing the information of near-field sources.

Step 4. Implement the EVD of $\mathbf{R}_{n}$ and then construct the polynomial (21) to obtain the roots of it. Based on (23), the DOA estimation of near-field sources is acquired.

Step 5. Choose the number of subregions $\beta$ and calculate the orthogonal matrix of signal subspace $\mathbf{T}_{k}$ by (38). Based on the matrix $\mathbf{T}_{k}$, the matrix $\mathbf{Q}$ is obtained by (39). Finally, the matrix $\mathbf{U}_{\text {new }}$ is obtained by (41).

Step 6. Construct $\mathbf{P}_{\text {new }}(r)$ by (36) and search only over sector $\varphi_{1}$ to obtain the spectral peak $\widehat{r}_{1}$. Use (26) to compute the other candidate range information by

$$
\widehat{r}_{k}=\frac{\widehat{r}_{1}}{1+\widehat{r}_{1}(1-m)}, \quad k=2,3, \ldots, \beta .
$$

Step 7. Select the true range information among the $\widehat{r}_{i}, i=$ $1,2, \ldots, \beta$ by maximizing $1 /\left\|\widehat{\mathbf{U}}_{N n}^{H} \mathbf{a}(\theta, r)\right\|$.

\section{Performance Analysis}

In this section, we analyze the computational complexity and the aperture freedom of the proposed algorithm and compare it with that of TSMUSIC algorithm.

5.1. Complexity Analysis. We only consider the majority parts of the computational complexity such as multiplication needed in constructing the cumulant matrix, EDV, and spectral search.

(1) The TSMUSIC algorithm requires constructing two different dimensional four order cumulant matrices, one is a $(2 N+1) \times(2 N+1)$ matrix and the other is $(4 N+$ $1) \times(4 N+1)$ matrix, while the proposed algorithm only constructs one $(2 N+1) \times(2 N+1)$ covariance matrix and one oblique projection operation with $(2 N+1) \times(2 N+1)$ dimension. 
(2) When estimating the information of the range, the TSMUSIC algorithm estimates the distance of the farfield sources and the near-field sources simultaneously. If there are $L$ sources, the traditional algorithms need $L$ times estimation for range. Since the farfield sources and near-field sources are estimated at two different stages in the proposed algorithm, the proposed algorithm only needs estimating the range of near-field sources. The time of $1-\mathrm{D}$ search will be less than $L$. The more number of far-field sources is, the less the time the proposed algorithm costs comparing to the other algorithms.

(3) Meanwhile, the proposed algorithm only needs to search the part of Fresnel area instead of the whole Fresnel area with the idea of compress, further leading to reducing the computational complexity ideally.

5.2. Aperture Freedom Analysis. The total number of the mix sources which can be estimated by the proposed algorithm is $2 N$ which is less than the number of arrays. The number of the near-field sources which can be estimated by the proposed algorithm is associated with the the number of subregions $\beta$ : the larger the value of $\beta$ is, the less the number of sources is. The relationship between the value $\beta$ and the number of nearfield sources $k_{n}$ is given as follows:

$$
\begin{gathered}
k_{n} \leq\left\lfloor\frac{2 N}{\beta}\right\rfloor \\
k_{f} \leq 2 N-k_{n},
\end{gathered}
$$

where $\lfloor\cdot\rfloor$ is under the integral function and $k_{f}$ is the number of the far-field sources which can be stimated by the proposed algorithm.

\section{Discussion}

The proposed algorithm can extend to a 3D array geometry. The detailed process is given as follows.

Consider three uniform linear arrays consisting of $2 \mathrm{~N}+1$ identical sensors which are located in the coordinate system $x$-axis, $y$-axis, and $z$-axis. The distance between the arrays is $d(d=\lambda / 4)$; it is depicted in Figure 4 and the regulation of angle expressed is also shown in Figure 4.

Based on the signal model in the literature [24], the signals which are received by three linear uniformly spaced arrays are given as follows:

$$
\begin{array}{r}
x_{i}(t)=\sum_{k=1}^{K_{n}} a_{n x_{i}}\left(\alpha_{k}, r_{k}\right) s_{n_{k}}(t)+\sum_{k=1}^{K_{f}} a_{f x_{i}}\left(\alpha_{k}\right) s_{f_{k}}(t)+n_{i}(t) \\
-N \leq i \leq N \\
y_{i}(t)=\sum_{k=1}^{K_{n}} a_{n y_{i}}\left(\beta_{k}, r_{k}\right) s_{n_{k}}(t)+\sum_{k=1}^{K_{f}} a_{f y_{i}}\left(\beta_{k}\right) s_{f_{k}}(t)+n_{i}(t) \\
-N \leq i \leq N
\end{array}
$$

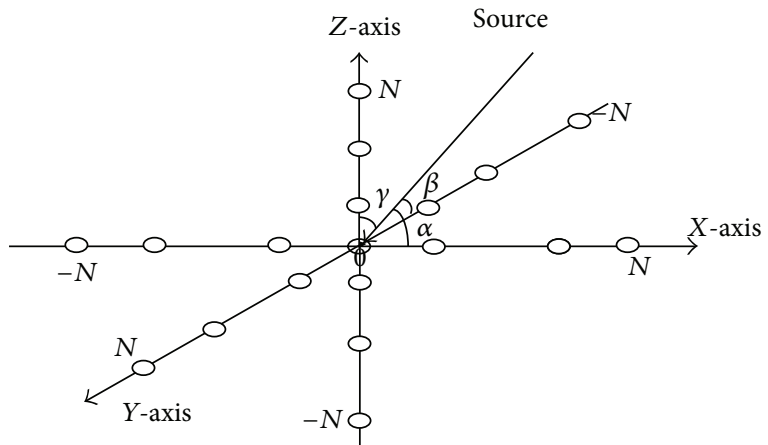

FIGURE 4: Symmetric uniform linear array configuration of 3D geometry.

$$
\begin{array}{r}
z_{i}(t)=\sum_{k=1}^{K_{n}} a_{n z_{i}}\left(\gamma_{k}, r_{k}\right) s_{n_{k}}(t)+\sum_{k=1}^{K_{f}} a_{f z_{i}}\left(\gamma_{k}\right) s_{f_{k}}(t)+n_{i}(t) \\
-N \leq i \leq N,
\end{array}
$$

where

$$
\begin{array}{r}
\mathbf{a}_{n x}\left(\alpha_{k}, r_{k}\right)=\left[\exp \left(j\left(-N \omega_{x k}+N^{2} \phi_{x k}\right)\right) \cdots\right. \\
\left.\exp \left(j\left(N \omega_{x k}+N^{2} \phi_{x k}\right)\right)\right]^{T} \\
\mathbf{a}_{f x}\left(\alpha_{k}\right)=\left[\exp \left(-j N \omega_{x k}\right) \cdots \exp \left(j N \omega_{x k}\right)\right]^{T} \\
\mathbf{a}_{n y}\left(\beta_{k}, r_{k}\right)=\left[\exp \left(j\left(-N \omega_{y k}+N^{2} \phi_{y k}\right)\right) \cdots\right. \\
\left.\exp \left(j\left(N \omega_{y k}+N^{2} \phi_{y k}\right)\right)\right]^{T} \\
\mathbf{a}_{f y}\left(\beta_{k}\right)=\left[\exp \left(-j N \omega_{y k}\right) \cdots \exp \left(j N \omega_{y k}\right)\right]^{T} \\
\mathbf{a}_{n z}\left(\gamma_{k}, r_{k}\right)=\left[\exp \left(j\left(-N \omega_{z k}+N^{2} \phi_{z k}\right)\right) \cdots\right. \\
\left.\exp \left(j\left(N \omega_{z k}+N^{2} \phi_{z k}\right)\right)\right]^{T} \\
\mathbf{a}_{f z}\left(\gamma_{k}\right)=\left[\exp \left(-j N \omega_{z k}\right) \cdots \exp \left(j N \omega_{z k}\right)\right]^{T}
\end{array}
$$

and $\omega_{x k}, \phi_{x k}, \omega_{y k}, \phi_{y k}, \omega_{z k}, \phi_{z k}$ are

$$
\begin{array}{ll}
\omega_{x k}=-\frac{2 \pi d \cos \left(\alpha_{k}\right)}{\lambda}, & \phi_{x k}=\frac{\pi d^{2} \sin \left(\alpha_{k}\right)^{2}}{\lambda r_{k}} \\
\omega_{y k}=-\frac{2 \pi d \cos \left(\beta_{k}\right)}{\lambda}, & \phi_{y k}=\frac{\pi d^{2} \sin \left(\beta_{k}\right)^{2}}{\lambda r_{k}} \\
\omega_{z k}=-\frac{2 \pi d \cos \left(\gamma_{k}\right)}{\lambda}, & \phi_{z k}=\frac{\pi d^{2} \sin \left(\gamma_{k}\right)^{2}}{\lambda r_{k}} .
\end{array}
$$

We use the proposed algorithm to process the data received by three orthogonal uniform linear arrays, respectively; the angle information of the far-field sources and the angle information and the range information of the nearfield sources were measured. It can be seen from a signal 
model that there is no direct link between the information of the three angles $(\alpha, \beta, \gamma)$, so we need to match angle to get the direction of the signal by some rules. The relationship between the three angles is shown as follows:

$$
(\cos \alpha)^{2}+(\cos \beta)^{2}+(\cos \gamma)^{2}=1 .
$$

Match process is finished by function (52).

Therefore the proposed algorithm in this paper can be extended to $3 \mathrm{D}$ space through the array model which is shown in Figure 4 and match process.

\section{Simulations}

In this section, several simulation results are presented to verify the performance of the proposed algorithm, which are compared with the TSMUSIC algorithm. In all experiences, we consider using a symmetric linear array consisting of $2 \mathrm{~N}+$ $1=17$ sensors with $d=\lambda / 4$ for the proposed algorithm and the compared methods. 500 Monte-Carlo runs are performed to obtain the experimental results, and the root mean square error (RMSE) is expressed as

$$
\text { RMSE }=\sqrt{\sum_{k=1}^{L} \sum_{n=1}^{M} \frac{\left(\widehat{\alpha}_{k}-\alpha_{k}\right)^{2}}{M L}},
$$

where $\widehat{\alpha}_{k}$ and $\alpha_{k}$ represent the estimation value and true value of the DOA $\theta_{k}$ or the range $r_{k}$, respectively.

In the first experiences, we consider four signals which contained two far-field sources and near-field sources. Two near-field sources are located at $\left\{\theta_{1}=20^{\circ}, r_{1}=4 \lambda\right\}$ and $\left\{\theta_{1}=\right.$ $\left.27^{\circ}, r_{1}=5 \lambda\right\}$. Two far-field sources are coming from $\left\{\theta_{3}=\right.$ $\left.40^{\circ}\right\},\left\{\theta_{4}=47^{\circ}\right\}$, and the number of subregions is $\beta=3$.

(1) RMSE and resolution probability versus SNR: the number of snapshots is fixed at 200, when SNR varies from 0 to $40 \mathrm{~dB}$; the resolution probability and the RMSE of DOA for far-field sources and near-field sources of the two algorithms are shown in Figures 5 and 6 , respectively. It obviously can be seen that the performance of the proposed algorithm is much better than the performance of TSMUSIC algorithm in all SNR. This is because the proposed algorithm estimates the two kinds of sources separately, which reduces the interaction between different kinds of signals. From Figure 7, it can be seen that the RMSE of the range information of the proposed algorithm outperforms the TSMUSIC algorithm in resolution probability and estimation accuracy in all SNR. This is because the accuracy of range information is affected by the accuracy of the DOA of near-field sources. The accuracy of DOA of the proposed algorithm is better than that of the TSMUSIC algorithm. So the accuracy of ROA of the proposed algorithm is better than that of the TSMUSIC algorithm.

(2) RMSE and resolution probability versus the snapshot number: the SNR is fixed at $20 \mathrm{~dB}$, when the snapshot number is varied from 50 to 500 . From Figures

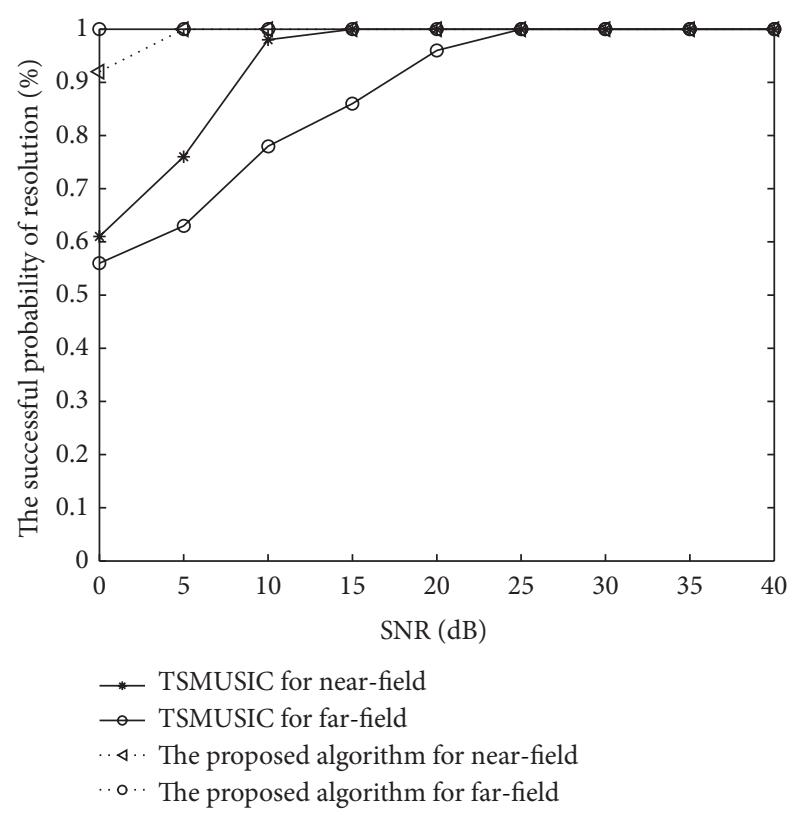

FIGURE 5: Resolution probability versus SNR.

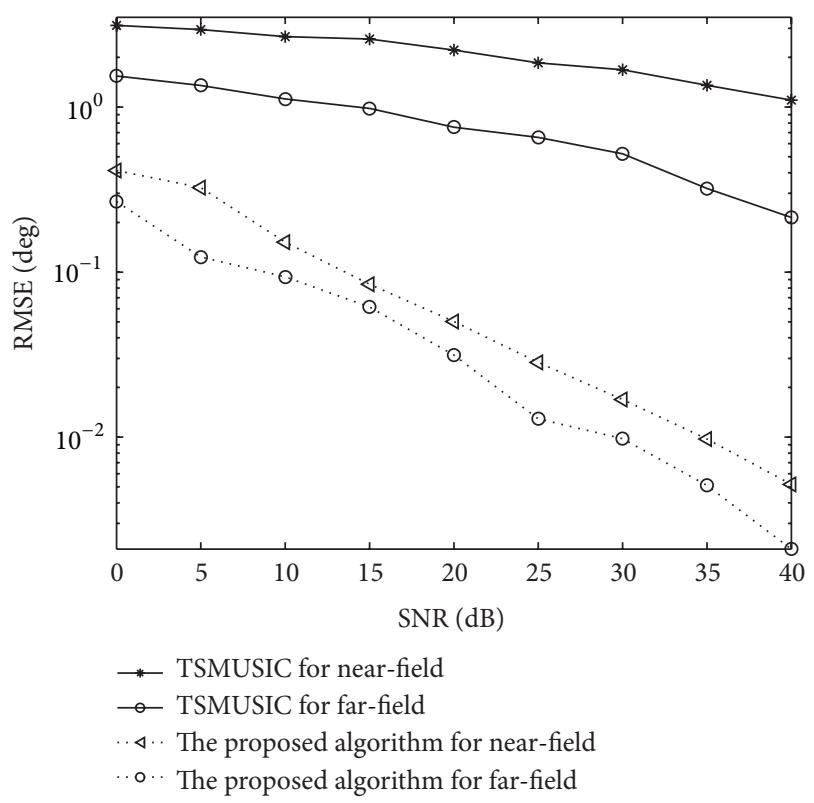

Figure 6: MSE of DOA estimation versus SNR.

8,9 , and 10 , it can be seen that the TSMUSIC algorithm is sensitive to the snapshot number. When the snapshot number is small, the performance of TSMUSIC is poor. However, the proposed algorithm is not sensitive to the snapshot number. The proposed algorithm outperforms the TSMUSIC algorithm in resolution probability and estimation accuracy for the same snapshot number.

In the second experiences, we consider four signals which contained two far-field sources and near-field sources, while the position of the sources is varied. The initial position of 
TABLE 1: The resolution probability versus angular gap.

\begin{tabular}{lcccc}
\hline The angle interval ${ }^{\circ}$ & $\begin{array}{c}\text { The proposed algorithm } \\
\text { for near-field source }\end{array}$ & $\begin{array}{c}\text { The proposed algorithm } \\
\text { for far-field source }\end{array}$ & $\begin{array}{c}\text { The TSMUSIC for far-field } \\
\text { source }\end{array}$ & $\begin{array}{c}\text { The TSMUSIC for } \\
\text { near-field source }\end{array}$ \\
\hline 11 & $100 \%$ & $100 \%$ & $91 \%$ & $95 \%$ \\
9 & $100 \%$ & $100 \%$ & $89 \%$ & $93 \%$ \\
7 & $100 \%$ & $100 \%$ & $73 \%$ & $82 \%$ \\
5 & $94 \%$ & $100 \%$ & $54 \%$ & $93 \%$ \\
3 & $90 \%$ & $100 \%$ & $3 \%$ & $0 \%$ \\
1 & $87 \%$ & $100 \%$ & $0 \%$ & $9 \%$ \\
\hline
\end{tabular}

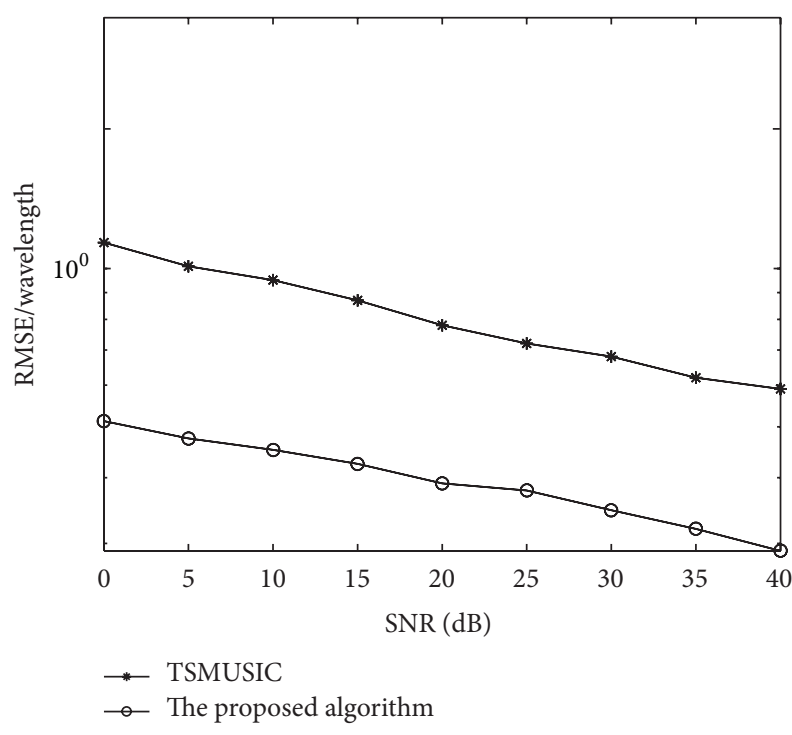

FIGURE 7: MSE of range estimation versus SNR.

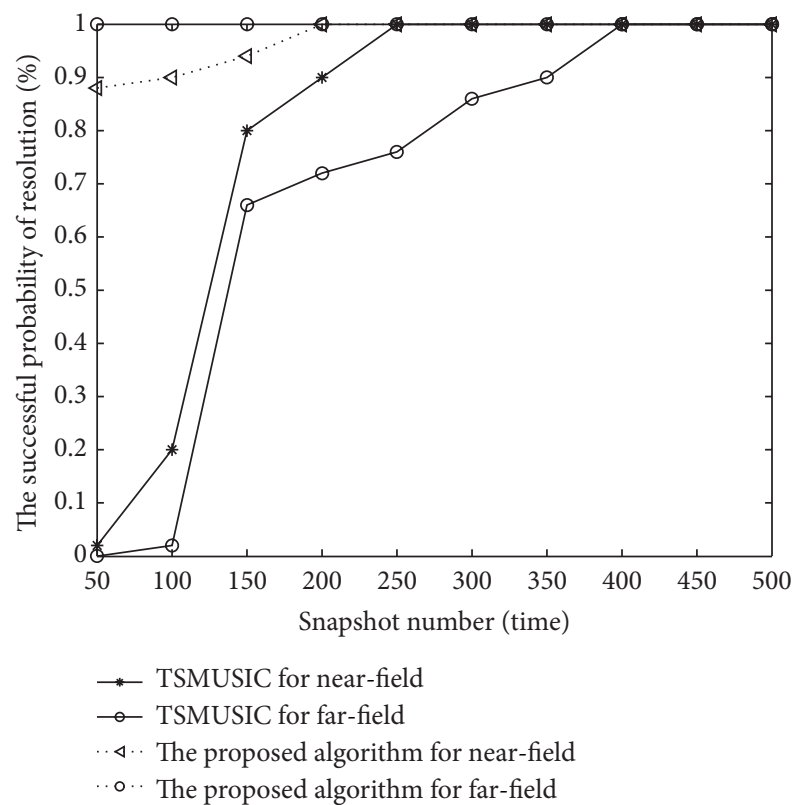

FIGURE 8: Resolution probability versus snapshots.

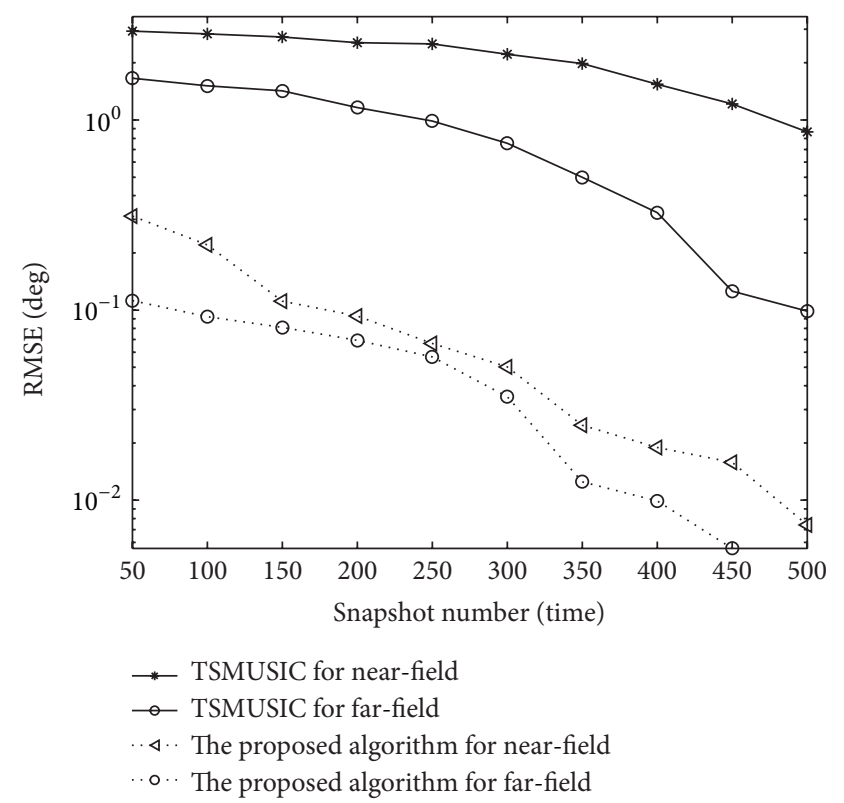

FIGURE 9: MSE of DOA estimation versus snapshots.

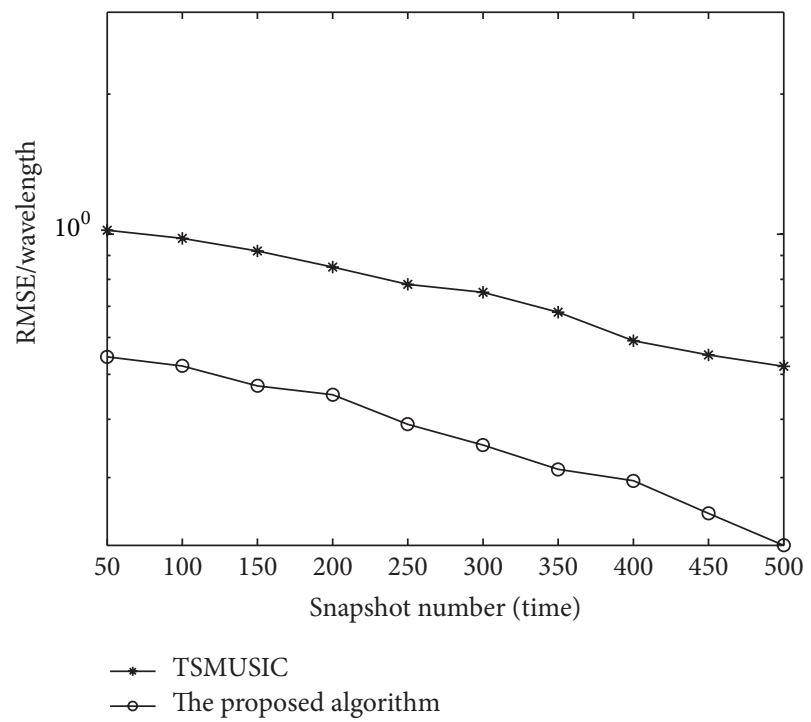

FIGURE 10: MSE of range estimation versus snapshots. 
the near-field sources is located at $\left\{\theta_{1}=20^{\circ}, r_{1}=4 \lambda\right\}$ and $\left\{\theta_{2}=31^{\circ}, r_{2}=5 \lambda\right\}$, and the initial position of far-field sources is located at $\left\{\theta_{3}=40^{\circ}\right\},\left\{\theta_{4}=51^{\circ}\right\}$.

(1) The resolution probability versus angular gap: the SNR is fixed at $10 \mathrm{~dB}$, the number of snapshot is fixed at 300 , the value of $\theta_{1}$ and $\theta_{3}$ is fixed. The value of $\theta_{2}$ is changed from $31^{\circ}$ to $21^{\circ}$, and the value of $\theta_{4}$ is changed from $51^{\circ}$ to $41^{\circ}$. From the Table 1 , it is clearly that the ability of estimating the closed-space sources of the proposed algorithm is much better than that of the TSMUSIC algorithm. The proposed algorithm uses the independent analysis methods for near-field sources and far-field sources, because they are estimated separately and each estimate is using all the data. Therefore, it is equivalent to reducing the number of sources, and the sensor noise power is estimated and eliminated from the covariance. So it is possible to estimate closely-space sources with high resolution probability and estimation accuracy.

(2) The resolution probability versus angular gap between the near-field source and far-field source. The SNR is fixed at $10 \mathrm{~dB}$ and the number of snapshots is fixed at 300 . The position of the near-field sources is located at $\left\{\theta_{1}=20^{\circ}, \gamma_{1}=4 \lambda\right\}$ and $\left\{\theta_{2}=\right.$ $\left.25^{\circ}, \gamma_{1}=5 \lambda\right\}$ and the position of far-field sources is located at $\left\{\theta_{3}=26^{\circ}\right\},\left\{\theta_{4}=31^{\circ}\right\}$. In order to distinguish the estimation performance of the two algorithms. The proposed algorithm uses the peak search instead of polynomial rooting. From Figure 12, it is clearly seen that the TSMUSIC algorithm cannot distinguish the angle $\theta_{2}$ of near-field source and the angle $\theta_{3}$ of the far-field sources, because of close space between the two sources. From the Figure 11, it is clearly seen that the proposed algorithm can easily distinguish the four sources, since the proposed algorithm estimates the near-field sources and farfield sources at different stages, which can effectively prevent mutual interference between the different kinds of sources (Figure 11).

(3) Calculate the simulation time of the proposed algorithm and the TSMUSIC algorithm. We use a MATLAB 7.11 to compare the simulation time with different algorithms in Table 2. We assume that the parameters of mixed sources are the same with the second experience. The SNR is fixed at $15 \mathrm{~dB}$. The number of near-field sources is fixed, the number of far-field sources is changed from 2 to 6 , and the number of subregions $\beta=2,3,4$. From Table 2, it is clear that the running time of the TSMUSIC algorithm increases with the increase of the number of far-field sources. However, the running time of the proposed algorithm is almost not changed. This is because the proposed algorithm does not need to estimate the range information of far-field sources, while the TSMUSIC algorithm needs. In the meanwhile, it can be seen that the larger $\beta$ is, the less time the proposed algorithm uses. The simulation result is identical to the theoretical analysis.
TABLE 2: The cost time versus the number of far-field sources.

\begin{tabular}{cccccc}
\hline \multicolumn{2}{c}{ The number of far-field sources } & 2 & 3 & 4 & 5 \\
\hline \multirow{4}{*}{ The proposed algorithm } & $\beta=2$ & 1.09 & 1.05 & 1.04 & 1.05 \\
& $\beta=3$ & 0.66 & 0.67 & 0.6 & 0.64 \\
& $\beta=4$ & 0.34 & 0.3 & 0.32 & 0.32 \\
\hline \multicolumn{2}{c}{ The TSMUSIC algorithm } & 2.59 & 3.36 & 4.12 & 4.82 \\
\hline
\end{tabular}

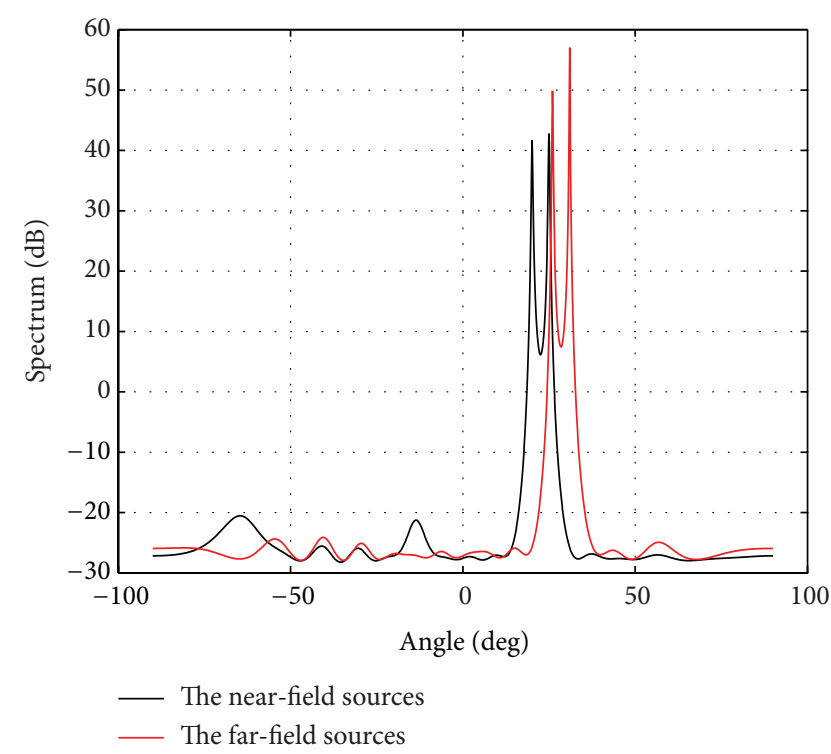

FIGURE 11: The spatial spectrum of the proposed algorithm for closely spaced sources.

\section{Conclusion}

In this paper, oblique projector technology is applied to estimate the mixed sources locations; thus the two different kinds of sources are estimated at two different stages. Utilizing the symmetric antenna structure, the polynomial is constructed and the roots of this polynomial are obtained, which are the DOAs of the near-field sources. Searching areas is focusing on part of Fresnel area instead of the whole Fresnel area with the idea of compress. Simulation result shows that the proposed algorithm has lower computation complexity, and it can solve the closely spaced signals and improve accuracy of estimating DOA and range. In the meanwhile, the proposed algorithm requires neither multidimensional searching nor high-order statistics computations calculation. In the future, we will focus on the mixed sources DOA estimation based on conformal array $[2,25,26]$.

\section{Appendices}

\section{A. Proof of the Equation (37)}

Based on the theory that the space spanned by steering vector is the same with the space spanned by signal subspace, we have

$$
\operatorname{span}\left(\mathbf{a}_{n}\left(\theta_{1}, r_{1}\right), \ldots, \mathbf{a}_{n}\left(\theta_{K_{n}}, r_{K_{n}}\right)\right)=\operatorname{span}\left(\mathbf{l}_{1}, \ldots, \mathbf{l}_{K_{n}}\right) .
$$




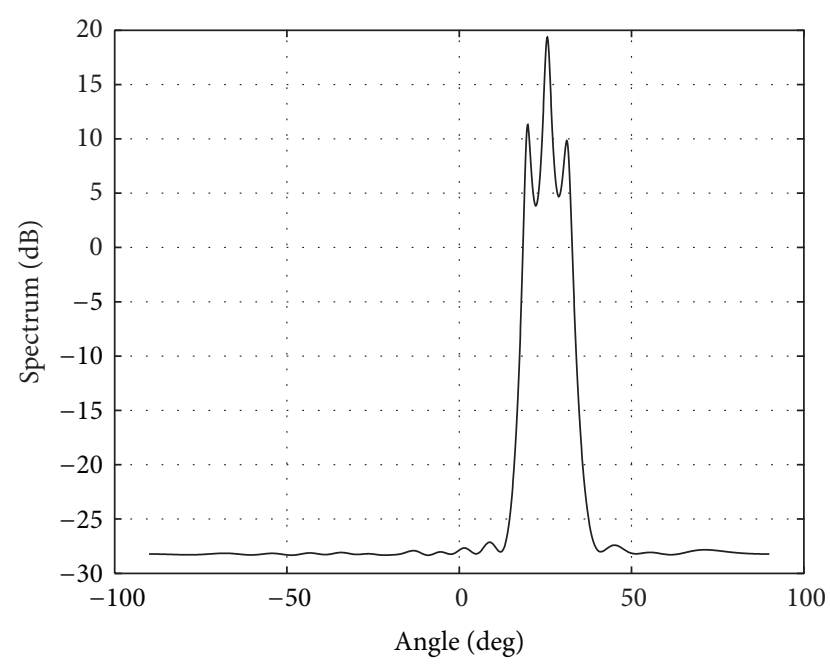

— The TSMUSIC algorithm

FIGURE 12: The spatial spectrum of the TSMUSIC algorithm for closely spaced sources.

Assuming that $r_{m} \in \psi_{m} m=1,2 \cdots \beta$ is the range information of the $m$ th source, according to (A.1), we have

$$
\mathbf{a}_{n}\left(\theta_{m}, r_{m}\right)=\left(\mathbf{l}_{1}, \ldots, \mathbf{l}_{K_{n}}\right) \mathbf{G}
$$

where $\mathbf{l}_{i}$ is the $i$ th column of signal subspace $\mathbf{L}$.

Substituting (A.2) into (28), we have

$$
\begin{aligned}
\chi_{k} & \odot \mathbf{a}_{n}\left(\theta_{m}, r_{m}\right) \\
& =\boldsymbol{\chi}_{k} \odot\left(\mathbf{l}_{1}, \ldots, \mathbf{l}_{K_{n}}\right) \mathbf{G} \longrightarrow \mathbf{a}_{n}\left(\theta_{m}, r_{k}\right) \\
& =\left(\mathbf{l}_{1 k}, \ldots, \mathbf{l}_{K_{n} k}\right) \mathbf{G},
\end{aligned}
$$

where $\mathbf{l}_{i, k}=\chi_{k}^{*} \odot \mathbf{l}_{i}$ is the $i$ th column of $\mathbf{S}_{k}$.

The expression of signal-like subspace cluster is expressed as follows:

$$
\begin{aligned}
\mathbf{L}_{k} & =\left[\mathbf{l}_{1, k}, \mathbf{l}_{2, k}, \ldots, \mathbf{l}_{K_{n}, k}\right] \\
& =\left[\boldsymbol{\chi}_{k}^{*} \odot \mathbf{l}_{1}, \boldsymbol{\chi}_{k}^{*} \odot \mathbf{l}_{2}, \ldots, \boldsymbol{\chi}_{k}^{*} \odot \mathbf{l}_{K_{n}}\right] \\
& =[\underbrace{\boldsymbol{x}_{k}^{*}, \boldsymbol{\chi}_{k}^{*}, \ldots, \boldsymbol{\chi}_{k}^{*}}_{K_{n}}] \odot\left[\mathbf{l}_{1}, \mathbf{l}_{2}, \ldots, \mathbf{l}_{K_{n}}\right], \quad k \in[1, \ldots, \beta] .
\end{aligned}
$$

\section{B. Proof of the Equation (40)}

It follows from (37) that $(i, j)$ th element of $\mathbf{P}_{k} \mathbf{P}_{k}^{H}$ is given by

$$
\begin{aligned}
\left(\mathbf{L}_{k} \mathbf{L}_{k}^{H}\right)_{i, j} & =\left(\chi_{k, i}^{*} \mathbf{l}_{i}\right)^{H}\left(\chi_{k, j} \mathbf{l}_{j}\right)=\left(\chi_{k, i}^{*} \chi_{k, j}\right) \mathbf{l}_{i}^{H} \mathbf{l}_{j} \\
& =\left(\chi_{k, i}^{*} \chi_{k, j}\right)\left(\mathbf{L}_{1} \mathbf{L}_{1}^{H}\right)_{i, j},
\end{aligned}
$$

where $\mathbf{l}_{i}$ and $\mathbf{l}_{j}$ are the $i$ th and the $j$ th row of $\mathbf{L}_{1}$, respectively:

$$
\mathbf{L}_{k} \mathbf{L}_{k}^{H}=\left(\boldsymbol{\chi}_{k}^{*} \boldsymbol{\chi}_{k}^{T}\right) \odot\left(\mathbf{L}_{1} \mathbf{L}_{1}^{H}\right)
$$

Similarly we can prove that

$$
\mathbf{U}_{k} \mathbf{U}_{k}^{H}=\left(\boldsymbol{\chi}_{k}^{*} \boldsymbol{\chi}_{k}^{T}\right) \odot\left(\mathbf{U}_{1} \mathbf{U}_{1}^{H}\right) .
$$

Thus it follows from (B.2) and (B.3) that

$$
\begin{aligned}
\mathbf{L}_{k} \mathbf{L}_{k}^{H}+\mathbf{U}_{k} \mathbf{U}_{k}^{H}= & \left(\boldsymbol{\chi}_{k}^{*} \boldsymbol{\chi}_{k}^{T}\right) \odot\left(\mathbf{U}_{1} \mathbf{U}_{1}^{H}+\mathbf{L}_{1} \mathbf{L}_{1}^{H}\right) \\
= & \left(\boldsymbol{\chi}_{k}^{*} \boldsymbol{\chi}_{k}^{T}\right) \odot \mathbf{I}=\mathbf{I} . \\
& \mathbf{P}_{k}=\mathbf{I}-\mathbf{U}_{k} \mathbf{U}_{k}^{H} .
\end{aligned}
$$

Substituting (B.5) into (38), we have

$$
\mathbf{Q}=\sum_{k=1}^{\beta} \mathbf{T}_{k}=\sum_{k=1}^{\beta} \mathbf{I}-\mathbf{U}_{k} \mathbf{U}_{k}^{H}=\beta \mathbf{I}-\sum_{k=1}^{\beta} \mathbf{U}_{k} \mathbf{U}_{k}^{H} .
$$

Suppose $\delta \in \operatorname{span}\left(\mathbf{U}_{\text {new }}\right)$; then we have $\delta \in \operatorname{span}\left(\mathbf{U}_{k}\right)$, $k=1, \ldots, \beta$. Since $\mathbf{U}_{k} \mathbf{U}_{k}^{H}$ is the orthogonal projection onto $\operatorname{span}\left(\mathbf{U}_{k}\right)$, we have $\delta=\mathbf{U}_{k} \mathbf{U}_{k}^{H} \delta$, which implies that $\|(\mathbf{I}-$ $\left.\mathbf{U}_{k} \mathbf{U}_{k}^{H}\right) \delta \|=0$. Therefore, we obtain

$$
\|\mathbf{Q} \delta\|=\left\|\left(\beta \mathbf{I}-\sum_{k=1}^{\beta} \mathbf{U}_{k} \mathbf{U}_{k}^{H}\right) \delta\right\| \leq \sum_{k=1}^{\beta}\left\|\left(\mathbf{I}-\mathbf{U}_{k} \mathbf{U}_{k}^{H}\right) \delta\right\|=0 .
$$

Thus, we have $\mathbf{Q} \delta=0$, and, therefore, $\delta \in \operatorname{Null}(\mathbf{Q})$. Since $\delta$ is an arbitrary vector of $\operatorname{span}\left(\mathbf{U}_{\text {new }}\right)$, we have

$$
\operatorname{span}\left(\mathbf{U}_{\text {new }}\right) \subseteq \operatorname{Null}(\mathbf{Q}) .
$$

On the other hand, assuming that $\delta \in \operatorname{Null}(\mathbf{Q})$, we obtain

$$
\mathbf{Q} \delta=\left(\beta \mathbf{I}-\sum_{k=1}^{\beta} \mathbf{U}_{k} \mathbf{U}_{k}^{H}\right) \delta=\beta \delta-\sum_{k=1}^{\beta} \mathbf{U}_{k} \mathbf{U}_{k}^{H} \delta=0 .
$$

Hence, we have

$$
\beta\|\delta\|=\left\|\sum_{k=1}^{\beta} \mathbf{U}_{k} \mathbf{U}_{k}^{H} \delta\right\| \leq \sum_{k=1}^{\beta}\left\|\mathbf{U}_{k} \mathbf{U}_{k}^{H} \delta\right\| .
$$

Noting that $\mathbf{U}_{k} \mathbf{U}_{k}^{H} \delta$ is the projection $\delta$ onto $\operatorname{span}\left(\mathbf{U}_{k}\right)$, we must have $\left\|\mathbf{U}_{k} \mathbf{U}_{k}^{H} \delta\right\| \leq\|\delta\|$. Therefore, we further obtain

$$
\beta\|\delta\| \leq \sum_{k=1}^{\beta}\left\|\mathbf{U}_{k} \mathbf{U}_{k}^{H} \delta\right\| \leq \sum_{k=1}^{\beta}\|\delta\|=\beta\|\delta\| .
$$

Therefore, we have $\delta=\mathbf{U}_{k} \mathbf{U}_{k}^{H} \delta$. This implies that $\delta=$ $\mathbf{U}_{k} \mathbf{U}_{k}^{H} \delta \in \operatorname{span}\left(\mathbf{U}_{\text {new }}\right)$. As $\delta$ is an arbitrary vector of $\operatorname{Null}(\mathbf{Q})$, we have

$$
\operatorname{Null}(\mathbf{Q}) \subseteq \operatorname{span}\left(\mathbf{U}_{\text {new }}\right) .
$$

It is implied by (B.8) and (B.12) that $\operatorname{span}\left(\mathbf{U}_{\text {new }}\right)=$ $\operatorname{Null}(\mathbf{Q})$, and the proof is completed.

\section{Conflict of Interests}

The authors declare that there is no conflict of interests regarding the publication of this paper. 


\section{Acknowledgments}

This work was supported in part by the National Science Foundation of China under Grant no. 61201410 and in part by the Fundamental Research Focused on Special Fund Project of the Central Universities (Program no. HEUCF140803).

\section{References}

[1] G. Han, A. Qian, C. Zhang, Y. Wang, and J. J. P. C. Rodrigues, "Localization algorithms in large-scale underwater acoustic sensor networks: a quantitative comparison," International Journal of Distributed Sensor Networks, vol. 2014, Article ID 379382, 11 pages, 2014.

[2] L.-T. Wan, L.-T. Liu, W.-J. Si, and Z.-X. Tian, "Joint estimation of 2D-DOA and frequency based on space-time matrix and conformal array," The Scientific World Journal, vol. 2013, Article ID 463828, 10 pages, 2013.

[3] G. Han, C. Zhang, L. Shu, J. J. P. Rodrigues, and J. Lloret, "A mobile anchor assisted localization algorithm based on regular hexagon in wireless sensor networks," The Scientific World Journal, vol. 2014, Article ID 219371, 13 pages, 2014.

[4] R. O. Schmidt, "Multiple emitter location and signal parameters estimation," IEEE Transactions on Antennas and Propagation, vol. 34, no. 1, pp. 276-280, 1986.

[5] X. Zhang and D. Xu, "Low-complexity ESPRIT-based DOA estimation for colocated MIMO radar using reduced-dimension transformation," Electronics Letters, vol. 47, no. 4, pp. 283-284, 2011.

[6] H. Krim and M. Viberg, "Two decades of array signal processing research: the parametric approach," IEEE Signal Processing Magazine, vol. 13, no. 4, pp. 67-94, 1996.

[7] Y.-D. Huang and M. Barkat, "Near-field multiple source localization by passive sensor array," IEEE Transactions on Antennas and Propagation, vol. 39, no. 7, pp. 968-975, 1991.

[8] J. Liang and D. Liu, "Passive localization of near-field sources using cumulant," IEEE Sensors Journal, vol. 9, no. 8, pp. 953960, 2009.

[9] N. Kabaoglu, H. A. Cirpan, E. Cekli, and S. Paker, "Deterministic maximum likelihood approach for 3-D near field source localization," International Journal of Electronics and Communications, vol. 57, no. 5, pp. 345-350, 2003.

[10] J. C. Chen, R. E. Hudson, and K. Yao, "Maximum-likelihood source localization and unknown sensor location estimation for wideband signals in the near-field," IEEE Transactions on Signal Processing, vol. 50, no. 8, pp. 1843-1854, 2002.

[11] D. Starer and A. Nehorai, "Passive localization on near-field sources by path following," IEEE Transactions on Signal Processing, vol. 42, no. 3, pp. 677-680, 1994.

[12] A. J. Weiss and B. Friedlander, "Range and bearing estimation using polynomial rooting," IEEE Journal of Oceanic Engineering, vol. 18, no. 2, pp. 130-137, 1993.

[13] N. Yuen and B. Friedlander, "Performance analysis of higher order ESPRIT for localization of near-field sources," IEEE Transactions on Signal Processing, vol. 46, no. 3, pp. 709-719, 1998.

[14] E. Grosicki, K. Abed-Meraim, and Y. Hua, "A weighted linear prediction method for near-field source localization," IEEE Transactions on Signal Processing, vol. 53, no. 10, pp. 3651-3660, 2005.
[15] W. Zhi and M. Y.-W. Chia, "Near-field source localization via symmetric subarrays," IEEE Signal Processing Letters, vol. 14, no. 6, pp. 409-412, 2007.

[16] D. Malioutov, M. Cetin, and A. S. Willsky, "A sparse signal reconstruction perspective for source localization with sensor arrays," IEEE Transactions on Signal Processing, vol. 53, no. 8, pp. 3010-3022, 2005.

[17] J. Liang and D. Liu, "Passive localization of mixed near-field and far-field sources using two-stage music algorithm," IEEE Transactions on Signal Processing, vol. 58, no. 1, pp. 108-120, 2010.

[18] B. Wang, J. Liu, and X. Sun, "Mixed sources localization based on sparse signal reconstruction," IEEE Signal Processing Letters, vol. 19, no. 8, pp. 487-490, 2012.

[19] B. Wang, Y. Zhao, and J. Liu, "Mixed-order MUSIC algorithm for localization of far-field and near-field sources," IEEE Signal Processing Letters, vol. 20, no. 4, pp. 311-314, 2013.

[20] X. Xu, Z. Ye, and J. Peng, "Method of direction-of-arrival estimation for uncorrelated, partially correlated and coherent sources," IET Microwaves, Antennas and Propagation, vol. 1, no. 4, pp. 949-954, 2007.

[21] J. Yin and T. Chen, "Direction-of-arrival estimation using a sparse representation of array covariance vectors," IEEE Transactions on Signal Processing, vol. 59, no. 9, pp. 4489-4493, 2011.

[22] F. Yan, M. Jin, and X. Qiao, "Low-complexity DOA estimation based on compressed MUSIC and its performance analysis," IEEE Transactions on Signal Processing, vol. 61, no. 8, pp. 19151930, 2013.

[23] X. Zhang, Matrix Analysis and Application, Tsinghua University Press, Beijing, China, 2004.

[24] Y.-D. Guo, H. Xie, Y.-S. Zhang, J. Gong, and D. Shen, "Localization for near-field targets based on virtual array of MIMO radar," Radar Sicence and Technology, vol. 10, no. 1, pp. 82-87, 2012.

[25] L. Wan, W. Si, L. Liu, Z. Tian, and N. Feng, "High accuracy 2D-DOA estimation for conformal array using PARAFAC," International Journal of Antennas and Propagation, vol. 2014, Article ID 394707, 14 pages, 2014.

[26] W. Si, L. Wan, L. Liu, and Z. Tian, "Fast estimation of frequency and 2-D doas for cylindrical conformal array antenna using state-space and propagator method," Progress in Electromagnetics Research, vol. 137, pp. 51-71, 2013. 


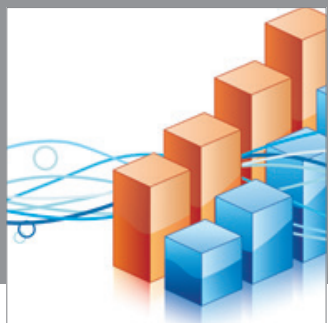

Advances in

Operations Research

mansans

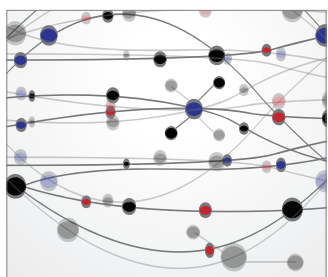

The Scientific World Journal
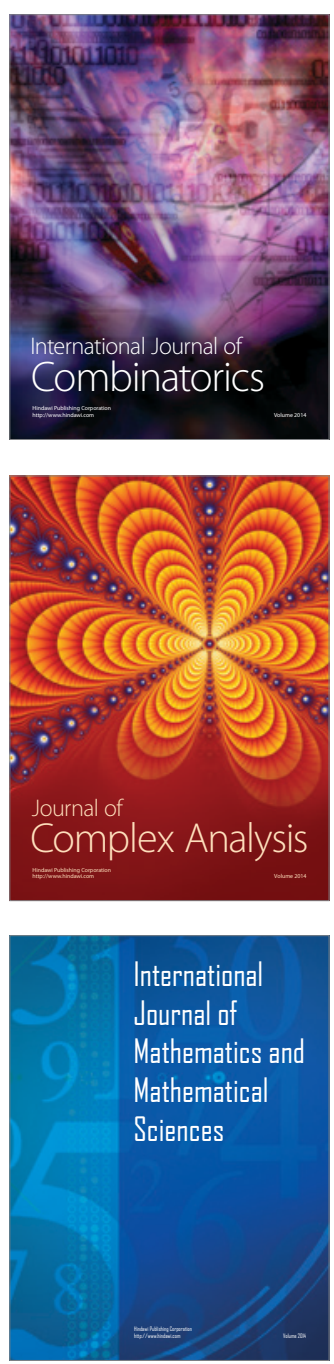
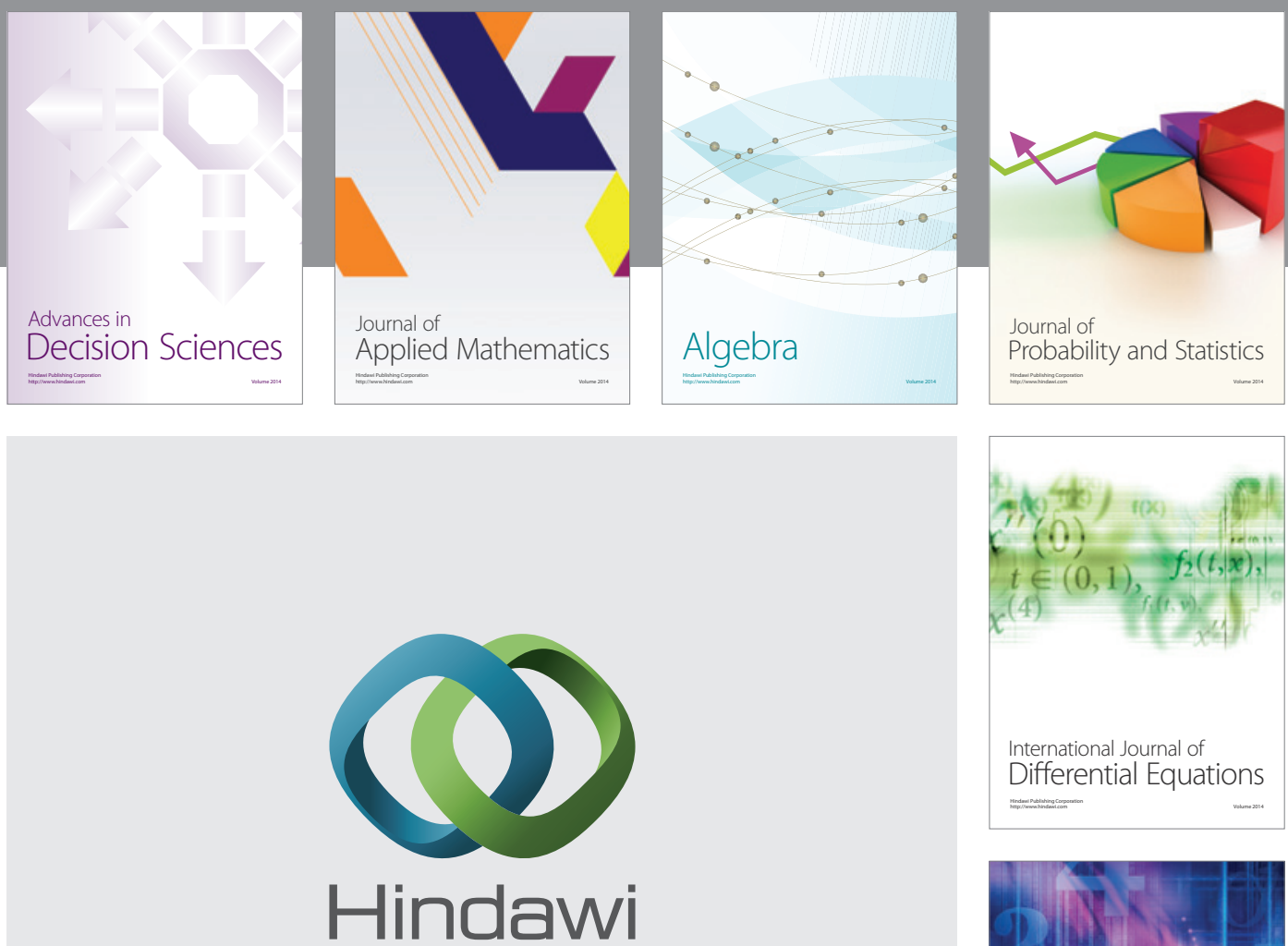

Submit your manuscripts at http://www.hindawi.com
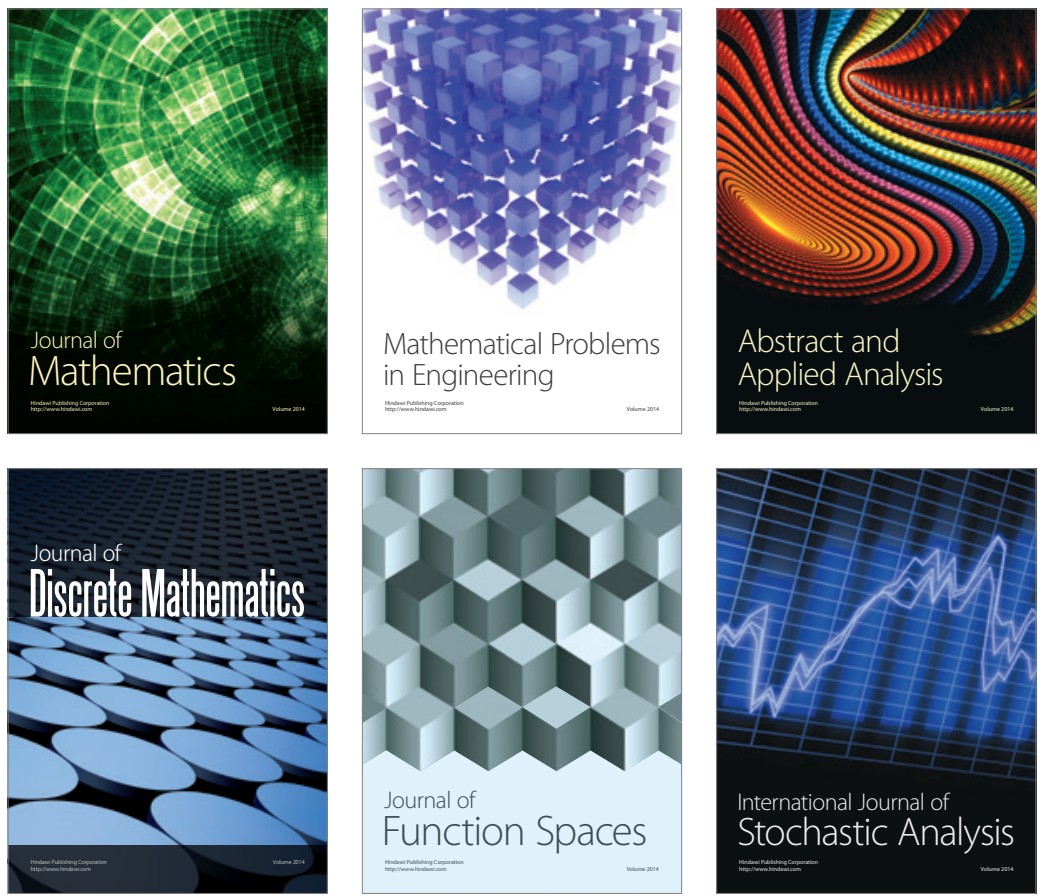

Journal of

Function Spaces

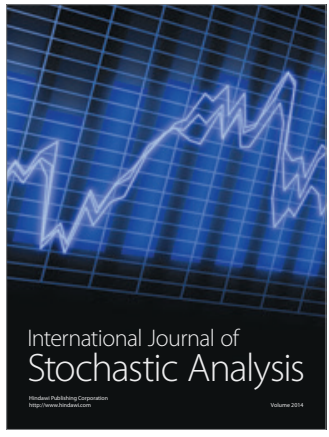

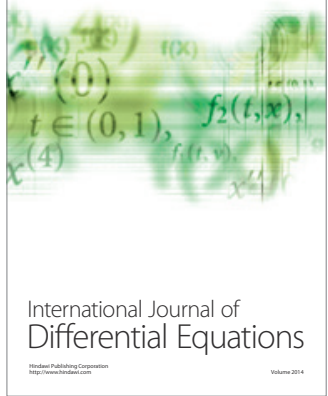
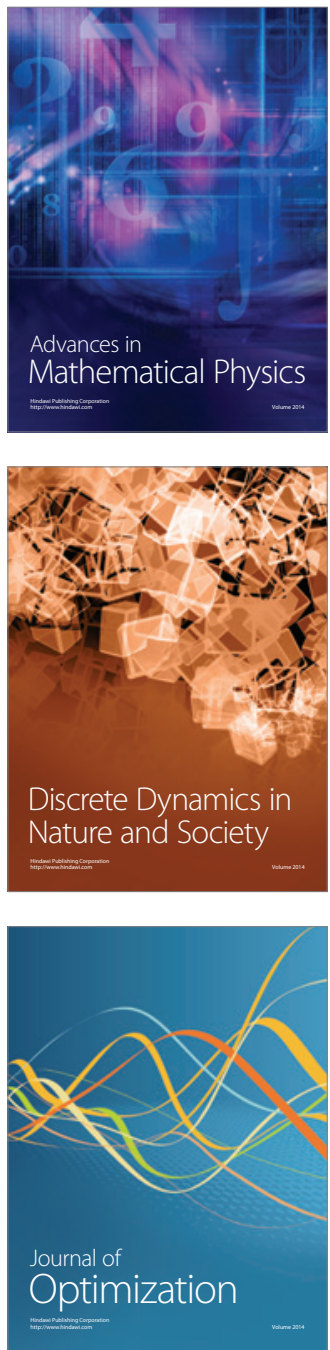